\title{
Ligand-induced dimerization of syndecan-3 at the cell surface
}

\author{
Evgeny Kulesskiy $^{1}$, Sarka Tumova ${ }^{2}$, Heikki Rauvala ${ }^{1}$ \\ ${ }^{1}$ Neuroscience Center, University of Helsinki, Helsinki, Finland \\ ${ }^{2}$ Multidisciplinary Cardiovascular Research Centre, Institute of Membrane \& Systems Biology, Leeds, UK \\ Email: evgeny.kulesskiy@helsinki.fi, heikki.rauvala@helsinki.fi
}

Received 6 March 2013; revised 2 May 2013; accepted 18 May 2013

Copyright (C) 2013 Evgeny Kulesskiy et al. This is an open access article distributed under the Creative Commons Attribution License, which permits unrestricted use, distribution, and reproduction in any medium, provided the original work is properly cited.

\begin{abstract}
Syndecan-3 (N-syndecan) is a transmembrane heparan sulfate proteoglycan abundantly expressed in developing brain. In addition to acting as a coreceptor, syndecan-3 acts as a signaling receptor upon binding of its ligand HB-GAM (heparin-binding growth-associated molecule; pleiotrophin), which activates the cortactin-src kinase signaling pathway. This leads to rapid neurite extension in neuronal cells, which makes syndecan- 3 as an interesting transmembrane receptor in neuronal development and regeneration. However, little is known about the signaling mechanism of syndecan-3. Here we have analyzed formation of ligand- $\mathrm{N}$-syndecan signaling complexes at the cell surface using fluorescence resonance energy transfer (FRET) and bioluminescence resonance energy transfer (BRET). We show that ligand binding leads to dimerization of syndecan- 3 at the cell surface. The dimerized syndecan-3 colocalizes with actin in the filopodia of cells. Several amino acid residues (K383, G392 and G396) in the transmembrane domain are shown to be important for the ligand-induced dimerization, whereas the cytosolic domain is not required for the dimerization.
\end{abstract}

Keywords: Syndecan-3; Dimerization; HB-GAM; Pleiotrophin; BRET; FRET

\section{INTRODUCTION}

Syndecan-3 (N-syndecan) is a transmembrane heparan sulfate proteoglycan (HSPG) expressed mainly in the nervous system [1]. Like the other members of the syndecan family, the ectodomain of syndecan-3 acts as a receptor or co-receptor in signaling processes for a broad range of ligands, including FGF-2 (fibroblast growth factor 2) [2], HB-EGF (heparin-binding EGF like growth factor) [3], and GDNF (glial cell-derived neurotrophic factor) family neurotrophic factors [4]. One of the main ligands is HB-GAM (pleiotrophin), a secretory extracellular matrix-associated protein that was initially isolated from rat brain as a neurite outgrowth-promoting factor $[5,6]$. In the case of HB-GAM, syndecan-3 acts as a signaling receptor. Binding of HB-GAM to syndecan-3 triggers formation of a signaling complex on the proteoglycan cytoplasmic tail, which leads to rapid neurite outgrowth in neuronal cells by activating the cortactinsrc kinase signaling pathway [3,7]. However, the mechanism of HB-GAM/syndecan-3 signaling remains largely unexplored.

Furthermore, syndecan-3 and HB-GAM have important physiological roles in limb skeletal development $[3,7,8]$ and in the control of feeding behaviour [9]. They play a crucial role in long-term potentiation (LTP) [10, 11], a long-lasting augmentation of synaptic strength that has been used as a cellular model for learning and memory.

Receptor dimerization is a crucial biological mechanism for receptor activation and regulation of signal transduction. Activation of receptor tyrosine kinases and other receptors that contain a single transmembrane domain is known to occur through dimerization in response to ligand binding [12-15]. Syndecans can form SDSresistant dimers and for syndecan 2 and 4 dimerization is implicated in receptor activation [16]. Although syndecan-3 dimerization was proposed to be involved in various signaling processes [17,18], its involvement in ligand binding and signal transduction has not been directly demonstrated before (Table S1).

Biochemical analyses, such as covalent cross-linking experiments or size exclusion chromatography can identify potential protein-protein interactions [19]. Such kind of approaches have been previously used for studying syndecan-3 dimerization (Table S1). Nevertheless, they 
are not suitable to study interactions in living cells. Fluorescence resonance energy transfer (FRET) microscopy adapted to different-colour fluorescent protein tags (e.g. CFP and YFP) [20] offers the opportunity to study the behaviour and spatial interaction of the tagged proteins in their natural environment in the living cell. Bioluminescence resonance energy transfer (BRET) is based on a similar principle but it utilizes bioluminescence rather than fluorescence and because of more robust signal provides means to simultaneously analyze multiple conditions, allowing simple and efficient screening.

We have used both FRET and BRET techniques to study syndecan-3 dimerization and show for the first time directly that syndecan-3 dimerizes at the surface of live cells upon binding of its ligand HB-GAM that has been shown to induce a cytoplasmic signalling complex that associates to syndecan-3. We have designed various syndecan-3 constructs to clarify the role of specific sequences in syndecan dimerization. A role of some amino acid residues in dimer formation has been previously assumed. However, previous research in this field has neglected to consider a full-length syndecan and dimerization that could be induced by ligand binding. All studies were done by gel filtration chromatography and SDS-PAGE using short transmembrane peptides or core proteins. Using single amino acid mutants in the transmembrane domain, we have now shown for the first time their requirement for ligand-induced dimerization. Moreover we show that the cytoplasmic domain of syndecan-3, albeit critical for cytoskeleton association and cytoplasmic signalling, is not required for dimerization.

\section{MATERIALS AND METHODS}

\subsection{DNA Constructs}

The pECFP-EYFP plasmid with 23-amino acid linker has been used as FRET positive control. This construct was made by insertion of $800 \mathrm{bp}$ AgeI-MfeI restriction fragment of pEYFP-C1 (Clontech) to pECFP-C1 plasmid in XmaI/MfeI restriction sites. Cotransfected $\mathrm{pECFP}$ and pEYFP vectors were used as negative controls.

Rat syndecan-3 cDNA was labeled by CFP/YFP sequence right after the transmembrane domain of the receptor in the cytosolic part (amino acids Tyr409/Arg410). Flexible amino acid linker sequence of 2, 5 or 6 amino acids was left between the receptor and the fluorophore (Figure S1(a)).

To express CFP/YFP-labeled syndecan-3 without cytoplasmic tail syndecan was tagged with CFP/YFP in a similar manner as described above for the full-length receptor. Stop codons were inserted just after the sequences encoding the CFP/YFP to delete the whole cytoplasmic tail (N-syn- $\Delta$ cyt-CFP and N-syn- $\Delta$ cyt-YFP).
pGFP $^{2}$-Rluc(h) (PerkinElmer) plasmid has been used as positive BRET control and also as a source for cloning all BRET constructs. $\mathrm{GFP}^{2} / \mathrm{Rluc}$ labeling of syndecan-3 and its mutants has been done in the same position as for CFP/YFP-constructs (Figure S1(b)).

Site-directed mutagenesis of syndecan-3 was done by using the following primers:

For K383A

CAGAAGAGCATACTAGAGCGGGCGGAGGTGCTCGTAGCTGTGAT

ATCACAGCTACGAGCACCTCCGCCCGCTCTAGTATGCTCTTCTG

For G392L, G396L

TAGCTGTGATCGTACTTGGCGTGGTGCTCGCCCTCTTCGCTGCCTT

AAGGCAGCGAAGAGGGCGAGCACCACGCCAAGTACGATCACAGCTA

\subsection{Cell Culture and Transfection}

HEK293T cells were grown in 35-mm glass bottom culture dishes (MatTek) in DMEM medium (Invitrogen), supplemented with 10\% FCS (Invitrogen), L-glutamine and penicillin-streptomycin at $37^{\circ} \mathrm{C}$ in $5 \% \mathrm{CO}_{2}$. Petri dishes were coated by poly-L-lysine $(20 \mu \mathrm{g} / \mathrm{ml})$, HBGAM $(50 \mu \mathrm{g} / \mathrm{ml})$, laminin $(10 \mu \mathrm{g} / \mathrm{ml})$ or BSA (100 $\mu \mathrm{g} / \mathrm{ml})$.

B-27 supplemented Neurobasal medium was used for culturing E17-18 hippocampal primary neurons isolated as described previously [4]. Hippocampal primary neurons were transfected by electroporation with $\mathrm{Nu}-$ cleofector Device (Amaxa) and Rat Neuron Nucleofector Kit using standard protocol (O-03 or G-13 programs).

Transfection of HEK293T cells was done by Fugene6 (Roche) and JetPei (Polyplus Transfection) transfection kits.

\subsection{Western Blot Analysis}

Whole cell lysate of HEK293T cells which expressed syndecan mutants were resolved by $4 \%$ - $15 \%$ SDSPAGE. Cells were deglycosylated at $37^{\circ} \mathrm{C}$ by $30 \mathrm{~min}$ incubation with heparinase I and III (H2519, H8891, Sigma-Aldrich) in PBS, $1 \mathrm{mM} \mathrm{CaCl}_{2}$. Western-blot semidry transfer was carried out in $10 \%$ methanol transfer buffer (30 mM Tris, $250 \mathrm{mM}$ glycine, 0,01\% SDS) at $15 \mathrm{~V}$ for $30 \mathrm{~min}$. Subsequent staining was done by anti syndecan-3 antibodies (sc-9496, Santa Cruz) with dilution $1: 200$.

\subsection{FRET Imaging and Analysis}

FRET imaging was performed essentially as described previously [3]. 12 - 24 hours after transfection with CFPand YFP-tagged constructs cells were washed with se- 
rum-free medium and incubated overnight without serum. Imaging was done at lower temperature $22^{\circ} \mathrm{C}-24^{\circ} \mathrm{C}$ to reduce the movements of cellular organelles [20]. Soluble HB-GAM (10 - $50 \mathrm{ng} / \mathrm{ml})$ or HB-GAM pre-coated $4.5 \mu \mathrm{m}$ polystyrene beads (Polysciences) were added to the culture dishes followed by incubation and the images were then acquired in different time scale.

FRET signal was quantified with three filter sets (Chroma): CFP channel (EX436/20, DCLP455, EM480/ 40); FRET channel (EX436/20, DCLP 455, EM535/30); YFP channel (EX500/20, DCLP515, EM535/30). Images were recorded in live transfected cells in CellR (Olympus) platform. The images were acquired in binning $2 \times$ 2 modes, to increase the signal-to-noise ratio, and 300 $500 \mathrm{~ms}$ integration times.

The background subtraction was made prior to the FRET calculations. All FRET images were corrected for crosstalk between CFP and YFP channels using linear spectral unmixing algorithm [20]. Corrected FRET $(\mathrm{Fc})$ was calculated with the following formula:

$$
\mathrm{Fc}=\mathrm{FRET}-a^{*} \mathrm{CFP}-b^{*} \mathrm{YFP}
$$

Correction factors were determined from separately expressed CFP or YFP cells: for each fluorophore the emission from the FRET channel was divided by the emission measured with either the CFP or YFP channels.

Correction factors $a$ and $b$ were found to be for HEK$293 T$ cells $0.39 \pm 0.009(\mathrm{n}=30)$ and $0.022 \pm 0.002(\mathrm{n}=$ 30) respectively, and for primary neurons $0.3975 \pm$ $0.0537(\mathrm{n}=12)$ and $0.0281 \pm 0.0053(\mathrm{n}=8)$.

The FRET-corrected images were transformed in pseudocolor mode to visualize high (shown in red) and low (shown in blue) values of FRET.

\subsection{BRET2 Assay}

HEK293T cells were harvested 24 or 48 hours after transfection with syndecan-3 constructs tagged with Rluc and GFP2 followed by washing with D-PBS buffer (PBS supplemented with $1 \mathrm{mM} \mathrm{CaCl}_{2}, 1 \mathrm{mM} \mathrm{MgCL}$ and $0.1 \%$ glucose). The cells were then transferred to pre-coated white 96-well microplate (OptiPlate-96, Perkin-Elmer) in the same buffer in $50 \mu 1 /$ well volume. To get suitable level of luminescence and to avoid non-specifically increasing BRET2 signal the total amount of cells was in the range 50,000 - 100,000 cells per well [22]. 96-well plates were pre-coated with different substrates: HBGAM $(10,100 \mu \mathrm{g} / \mathrm{ml})$, poly-L-lysine $(20 \mu \mathrm{g} / \mathrm{ml})$, BSA $(100 \mu \mathrm{g} / \mathrm{ml})$, b-FGF $(2 \mu \mathrm{g} / \mathrm{ml})$, GDNF $(20 \mu \mathrm{g} / \mathrm{ml})$, laminin $(10 \mu \mathrm{g} / \mathrm{ml})$ at $+4^{\circ} \mathrm{C}$ overnight. Cells were incubated on the plates for varying intervals ranging from 15 - 240 minutes. After that $50 \mu \mathrm{l}$ of $10 \mu \mathrm{M}$ DeepBlueC Coelenterazine substrate was added by dispenser module to each well followed by measurements of signal in
410/80 $\mathrm{nm}$ (Rluc) and 515/30 $\mathrm{nm}\left(\mathrm{GFP}^{2}\right)$ filters in VIC$\mathrm{TOR}^{3}$ Multilabel Plate Reader (Perkin-Elmer). BRET2 signal was calculated as a ratio of emission at $515 \mathrm{~nm}$ (minus background) to emission at $410 \mathrm{~nm}$ (minus background). Background is defined as a signal of nontransfected cells in the respective channel.

\subsection{Actin Staining and Fluorescent Imaging}

HEK293T cells expressing YFP fusion full-length and cytoplasmic tail lacking syndecan-3 (NS-YFP and Nsyn- $\Delta$ cyt-YFP) constructs were used in actin colocalization experiments. Transfected cells were transferred to plates with different pre-coated substrates and subsequently fixed in $4 \%$ formaldehyde and stained by phalloidin-rhodamin. Images were recorded in two channels: TRITC (EX540/25, DCLP560, EM605/55) and YFP (EX500/20, DCLP515, EM535/30).

\section{RESULTS AND DISCUSSION}

\subsection{FRET Shows Dimerization of Syndecan-3 at the Cell Surface}

HEK293T cells co-expressing two types of full-length syndecan-3 constructs (CFP and YFP fusion proteins) were plated on glass cover slips coated with different ligands. Cells grown on HB-GAM-coated surface (Figure 1(b)) show after 4 hours of incubation clearly higher FRET signal compared to BSA-coated controls (Figure 1(a)). Signal from cells on BSA surface could be explained by basal, ligand-independent syndecan-3 dimerization. Syndecan-3 lacking the C-terminal cytoplasmic part has similar levels of FRET signal (Figure 1(c)), showing that the cytoplasmic domain of syndecan-3 is not required for dimerization. This mutant stimulates filopodia formation and FRET signal is clearly visible in the cellular processes. Addition of soluble HB-GAM also stimulates syndecan-3 dimerization, as evidenced by the increase of FRET signal (Figure 1(c)).

We have repeated our FRET experiments in 2 weeks old hippocampal primary neurons. To provide sufficient expression levels for FRET measurement, neurons were transfected by electroporation with fluorescently labelled syndecan-3 constructs. To show ligand-dependent specificity of FRET signals, dimerization was induced by HB-GAM coated beads. The FRET signal accumulates in places where the beads contact hippocampal neurons (Figure 2(a)).

In FRET analysis it is critical to subtract all crosstalks between fluorophores. The absence of false positive values in negative controls confirm that the correction factors were calculated correctly while positive controls expressing pCFP-YFP show maximum FRET signal (Figures 1(e) and 2(b)). 


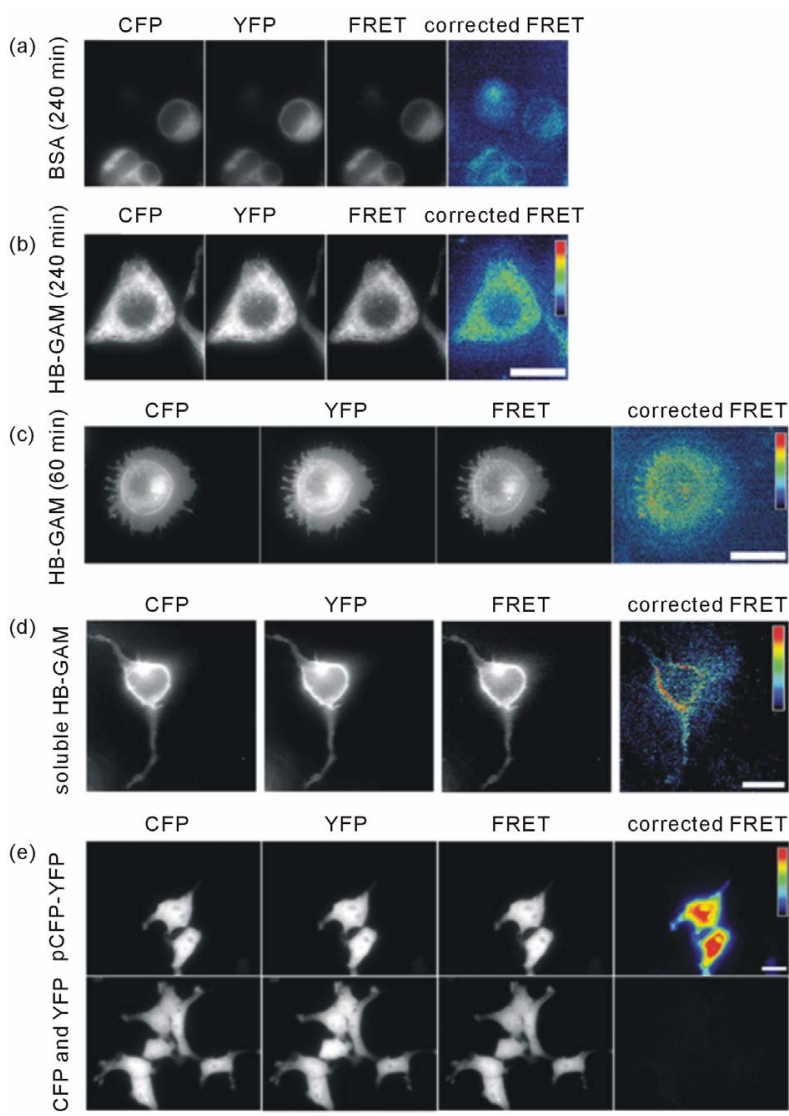

Figure 1. FRET images of HEK293T cells expressing syndecan-3 under different conditions. (a), (b) Cells plated on BSA (a) and HB-GAM (b) coated culture dishes after 4 hours of culturing. Full length syndecan-3 (NS-CFP/YFP-cd) constructs were used in this experiment. (c) Cells expressing a cytoplasmic tail deletion variant of syndecan-3 (NS-CFP/YFP- $\Delta \mathrm{cd}$ ) on HBGAM coated substrate after 60 minutes of incubation. (d) HEK293T cells expressing a full-length syndecan-3 after addition of soluble HB-GAM. (e) Control constructs expressed in HEK293T cells. Positive control cells expressed pCFP-YFP plasmid with 23 amino acid linker, for negative control, cells were cotransfected with pECFP and pEYFP vectors. FRET corrected images are shown in pseudocolor mode to transform the input data into a more visible output format. High values of FRET signal are shown in red and low values shown in blue. Bars- $10 \mu \mathrm{m}$.

\subsection{Ligand Screening by BRET Assay}

We have used the BRET assay for quantitative comparison of dimerization induced by different ligands. The BRET assay is widely used for studying ligand-induced conformational changes and/or dimerization of receptors as well as for searching of new ligands and new drug leads [23-25]. In our studies we applied the second generation of BRET assay, BRET2, based on using of GFP ${ }^{2}$ and DeepBlueC Coelenterazine substrate which give a lower background and much better separation of donor (RLuciferase construct) and acceptor (GFP2 construct) signals.

(a)

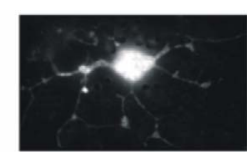

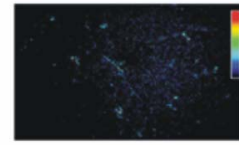

$0 \mathrm{~min}$

CFP

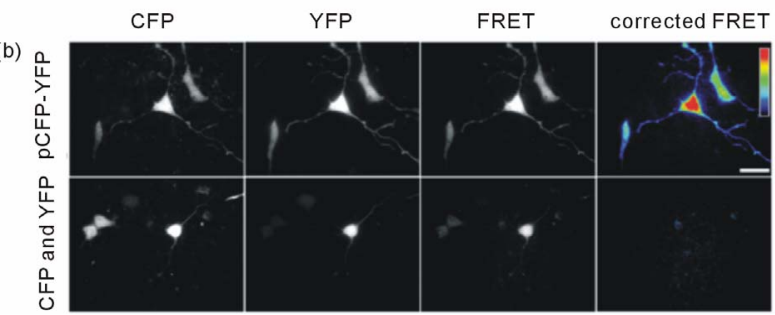

Figure 2. FRET images of 2 weeks old hippocampal neurons expressing fluorescently labeled syndecan-3 constructs after addition of HB-GAM coated beads. (a) Hippocampal neurons expressing FRET constructs after 10 and 20 min of incubation with HB-GAM-coated beads (stars). (b) Control constructs expressed in neurons. Negative and positive control cells were transfected as indicated in the legend for Figure 1(e). Bars-20 $\mu \mathrm{m}$.

Similar to the FRET analysis, the BRET2 assay shows that ligand-induced syndecan-3 dimerization occurs upon plating of cells on HB-GAM-coated plates. In addition, there is significant increase in BRET ratio for bFGFcoated wells (Figure 3(a)). Previously published data suggests that glial cell line-derived neurotrophic factor (GDNF) can interact with heparan sulphate proteoglycans on cell surface or in extracellular matrix and that heparan sulphates are required as co-receptors for GDNF signalling $[26,27]$. We have shown recently by FRET that GDNF induces syndecan-3 dimerization [4], therefore we also included GDNF in the current screening. GDNF induced dimerization to the same degree as the established ligand HB-GAM. All control proteins (BSA, laminin, poly-L-lysine) which were used in this assay were similar to the blank BRET-ratio values (Figure 3(a)).

BRET was used to assay the kinetics of changes in syndecan-3 dimerization upon HB-GAM binding. The highest signal was obtained after 15 minutes of incubation. The signal then decreased but was still detectable above background even up to $4 \mathrm{~h}$ (Figure 3(b)).

\subsection{BRET Assay of Transmembrane Domain Mutants}

It has been previously suggested that substitutions of some amino acid residues (K383, G392 and G396) from the transmembrane domain could inhibit syndecan-3 self-association in vitro [17]. Gel electrophoresis and size 


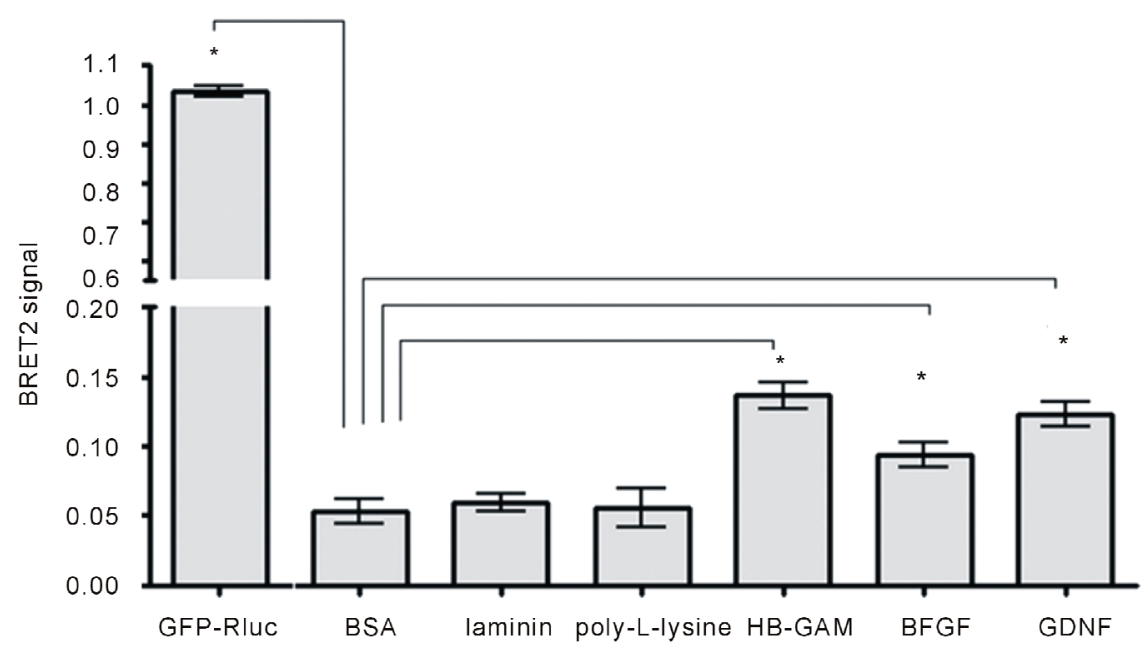

(a)

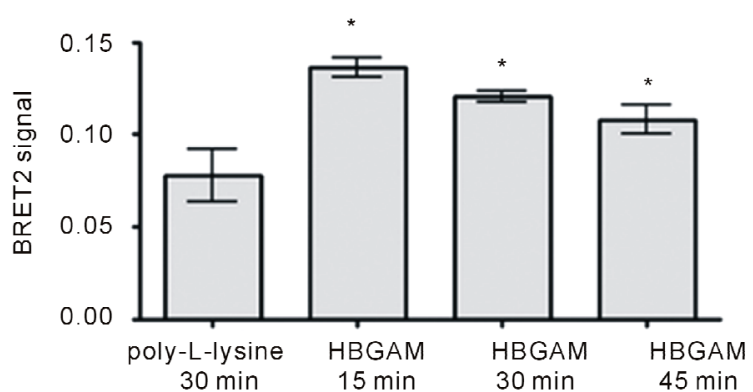

(b)

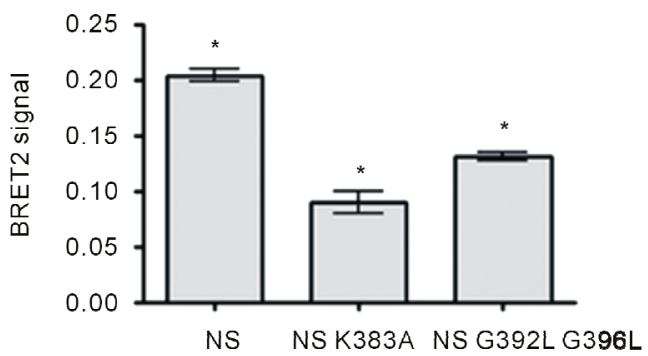

(c)

Figure 3. BRET2 analysis of ligand-induced dimerization of syndecan-3. (a) Screening of different ligands (24 hours after transfection with Rluciferase-syndecan-3 and GFP2-syndecan-3). Plates were pre-coated at the following concentrations: HB-GAM $(10 \mu \mathrm{g} / \mathrm{ml})$, poly-L-lysine $(20 \mu \mathrm{g} / \mathrm{ml})$, BSA $(100 \mu \mathrm{g} / \mathrm{ml})$, b-FGF $(2 \mu \mathrm{g} / \mathrm{ml})$, GDNF $(20 \mu \mathrm{g} / \mathrm{ml})$, laminin $(10 \mu \mathrm{g} / \mathrm{ml})$. Transfected cells were incubated on the plates for 15 minutes and BRET ratio measured. P-values lower than $0.05(\mathrm{n}=8)$ are indicated with asterisk. (b) Kinetics of HB-GAM-induced dimerization of syndecan-3 (24 hours after transfection). P-values lower than $0.05(\mathrm{n}=12)$ are indicated with asterisk. (c) Impaired dimerization of syndecan-3 transmembrane mutants. Cells were transfected with wild type or mutant syndecan-3 constructs for 48 hours to achieve sufficient level of expression for the mutants. After 15 min incubation with HB-GAM coated plates BRET ratio was measured showing significantly lower level of mutant dimerization compared to the wild-type protein. P-values lower than $0.05(\mathrm{n}=12)$ are indicated with asterisk.

exclusion chromatography of E. coli expressed MBPsyndecan-3 fusion proteins was used in these studies to demonstrate the residue involvement in dimerization but the role of ligand stimulation was not determined.

We therefore applied the BRET2 assay to test whether these amino acid residues are involved in ligand-induced dimerization in live cells, using K383A and G392L/ G396L mutants of syndecan-3 cloned in BRET-constructs. To check that all GFP/Rluc variants of the syndecan-3 mutants are expressed at similar levels, they were transfected to HEK293T cells and compared to syndecan-3 wild-type fusion proteins by western-blot analysis (Figure S2(a)). Correct localization was verified by fluorescent imaging of GFP-fused mutants and wildtype syndecan-3 (Figure S2(b)). All mutant proteins localized on the surface of cells similar to the wild type.
In addition, tiny networks of fluorescently labeled proteins were observed which may correspond to colocalization with the actin cytoskeleton as it was shown previously for syndecan-3 $[28,29]$ as well as for other syndecans [30-32].

Finally, the mutants and wild-type syndecan-3 were screened by BRET2 assay to check the role of K383, G392 and G396 amino acid residues in ligand-induced dimerization. The HEK293T cells were grown for $48 \mathrm{~h}$ after transfection to get sufficient level of expression. HB-GAM stimulated dimerization of wild type syndecan-3 but both mutant variants (K383A and G392L/ G396L) had statistically significantly lower BRET-ratio signals in the presence of HB-GAM (Figure 3(c)) as well as on poly-L-lysine, indicating their diminished ability to dimerize. 


\subsection{Syndecan-3-Actin Colocalization}

BRET and FRET experiments show that HB-GAM-induced dimerization of syndecan-3 occurs mainly within the first 20 minutes. This data corresponds well to syndecan - actin colocalization. HEK293T cells plated on HB-GAM show very high colocalization of syndecan and actin within the first 30 minutes (Figure 4(a)) compared to cells growth on BSA that do not show colocalization (Figure 4(d)). After $4 \mathrm{~h}$, colocalization can mainly be found in cellular processes (Figure 4(b)). The cytoplasmic tail deletion variant of syndecan-3 does not show any colocalization with actin (Figure 4(c)).

\section{CONCLUDING REMARKS}

Syndecans are generally suggested to function as coreceptors, sequestering growth factors in extracellular matrices and on the cell surface locations for presentation to signaling receptors. However, our previous studies have suggested that syndecan-3 may act as an independent signaling receptor activating the cortactin-src kinase pathway upon binding of HB-GAM [3,7].
It has been well established that many signaling receptors, such as tyrosine kinase receptors, dimerize at the cell surface upon ligand binding which is the key event to trigger signal transduction. The ability of syndecans to form (SDS-resistant) dimers has been documented and for syndecan- 2 and -4 , and the ability to form dimers has been linked to their activation [16,33]. We have elucidated in this study behavior of syndecan-3 at the cell surface upon HB-GAM binding using FRET and BRET to detect syndecan-3 dimerization in live cells. We demonstrate that syndecan-3 is clearly dimerized upon HB-GAM binding, resembling the behavior of wellknown signaling receptors such as transmembrane tyrosine kinases. Furthermore, mutant forms of syndecan-3 show that the transmembrane domain, but not the cytosolic domain, is required for ligand-induced dimerization. Time range of the observed dimerization, peaking at 15 minutes, agrees well with the formation of the kinase signaling complex on the syndecan-3 cytoplasmic tail, which was reported at $20 \mathrm{~min}$ after HB-GAM stimulation [7]. It is unclear at the present what is the exact role of dimerization in the signaling process. For trans- (a)

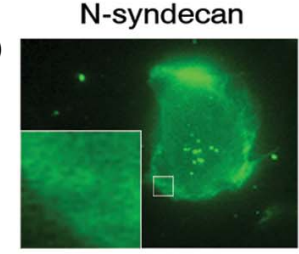

(b)

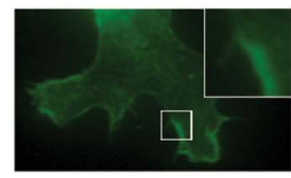

(c)

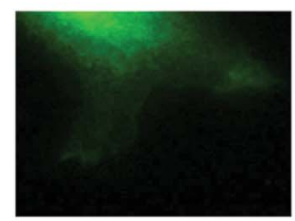

(d)

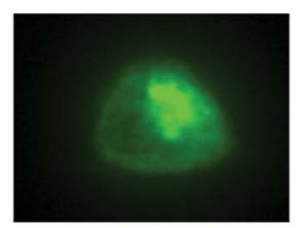

Actin
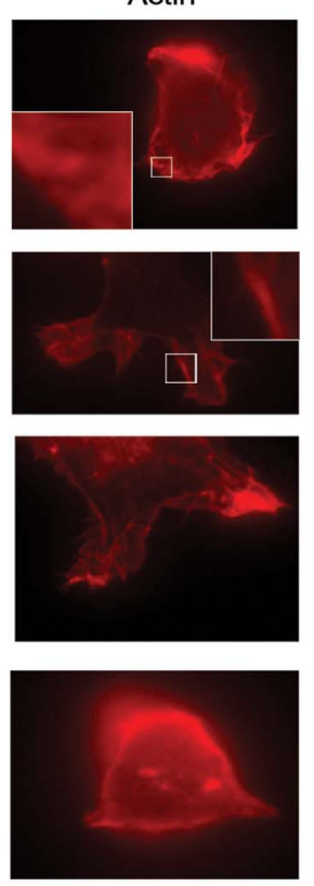

merge
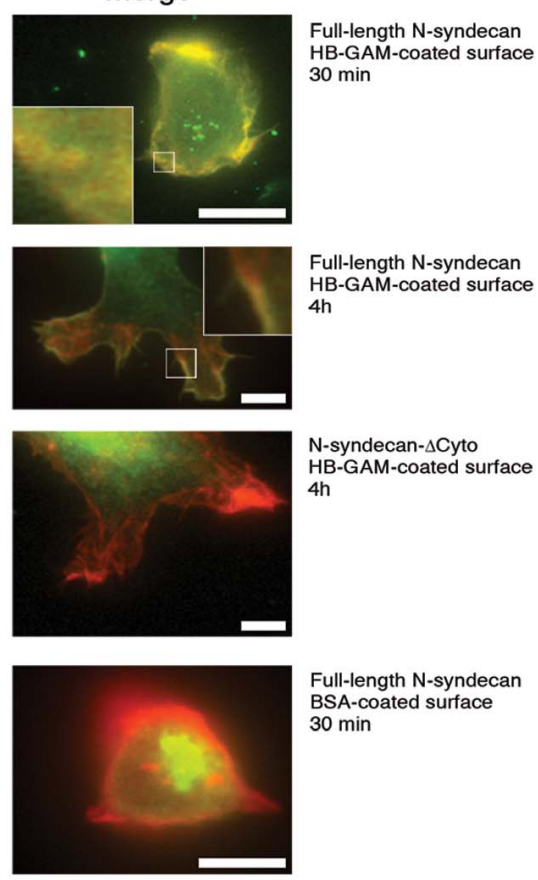

Full-length $\mathrm{N}$-syndecan HB-GAM-coated surface $30 \mathrm{~min}$

Figure 4. Syndecan-3-Actin colocalization. (a) HEK293T cells plated on HB-GAM-coated culture dishes for 30 minutes. YFP fusion full-length syndecan-3 (NS-YFP) construct colocalized with actin on the plasma membrane. Bars- $10 \mu \mathrm{m}$. (b) Cells transfected with the NS-YFP construct were plated on HB-GAM-coated culture dishes for 4 hours. Colocalization occurs mainly in the cellular processes. Bars $-2 \mu \mathrm{m}$. (c) Cytoplasmic tail deletion variant of syndecan-3 (NS-YFP- $\Delta$ cd) was expressed in HEK293T cells and cells were fixed and stained after 4 hours of incubation on HB-GAM-coated glass. Bars-2 $\mu \mathrm{m}$. (d) Cells expressing NS-YFP plated on BSA-coated cover slips after 30 minutes. Bars-10 $\mu \mathrm{m}$. A-D: Syndecan-3-YFP fusion protein is shown in green and actin filaments stained with Alexa Fluor 568 phalloidin are shown in red. Merged images are shown in the right column. 
membrane kinase receptors, dimerization brings the advantage of cross-phosphorylation. For syndecans, it could be clustering and forming a platform for facilitating interactions with cytoskeleton and multiple cytoplasmic signaling molecules.

\section{ACKNOWLEDGEMENTS}

We thank Seija Lehto for her excellent technical assistance. This work was supported by the Academy of Finland and the Sigrid Jusélius Foundation.

\section{REFERENCES}

[1] Carey, D.J., Evans, D.M., Stahl, R.C., Asundi, V.K., Conner, K.J., Garbes, P. and Cizmeci-Smith, G. (1992) Molecular cloning and characterization of N-syndecan, a novel transmembrane heparan sulfate proteoglycan. The Journal of Cell Biology, 117, 191-201. doi: $10.1083 /$ jcb.117.1.191

[2] Dealy, C.N., Seghatoleslami, M.R., Ferrari, D. and Kosher, R.A. (1997) FGF-stimulated outgrowth and proliferation of limb mesoderm is dependent on syndecan-3. Developmental Biology, 184, 343-350. doi:10.1006/dbio.1997.8525

[3] Hienola, A., Tumova, S., Kulesskiy, E. and Rauvala, H. (2006) N-syndecan deficiency impairs neural migration in brain. The Journal of Cell Biology, 174, 569-580. doi:10.1083/jcb.200602043

[4] Bespalov, M.M., Sidorova, Y.A., Tumova, S., AhonenBishopp, A., Magalhães, A.C., Kulesskiy, E., Paveliev, M., Rivera, C., Rauvala, H. and Saarma, M. (2011) Heparan sulfate proteoglycan syndecan-3 is a novel receptor for GDNF, neurturin, and artemin. The Journal of Cell Biology, 192, 153-169. doi:10.1083/jcb.201009136

[5] Rauvala, H. (1989) An 18-kd heparin-binding protein of developing brain that is distinct from fibroblast growth factors. The EMBO Journal, 8, 2933-2941.

[6] Li, Y.S., Milner, P.G., Chauhan, A.K., Watson, M.A., Hoffman, R.M., Kodner, C.M., Milbrandt, J. and Deuel, T.F. (1990) Cloning and expression of a developmentally regulated protein that induces mitogenic and neurite outgrowth activity. Science, 250, 1690-1694. doi:10.1126/science. 2270483

[7] Kinnunen, T., Kaksonen, M., Saarinen, J., Kalkkinen, N., Peng, H.B. and Rauvala, H. (1998) Cortactin-Src kinase signaling pathway is involved in $\mathrm{N}$-syndecan-dependent neurite outgrowth. The Journal of Biological Chemistry, 273, 10702-10708. doi:10.1074/jbc.273.17.10702

[8] Pacifici, M., Shimo, T., Gentili, C., Kirsch, T., Freeman, T.A., Enomoto-Iwamoto, M., Iwamoto, M. and Koyama, E. (2005) Syndecan-3: A cell-surface heparan sulfate proteoglycan important for chondrocyte proliferation and function during limb skeletogenesis. Journal of Bone and Mineral Metabolism, 23, 191-199. doi:10.1007/s00774-004-0584-1

[9] Reizes, O., Lincecum, J., Wang, Z., Goldberger, O., Huang, L., Kaksonen, M., Ahima, R., Hinkes, M.T.,
Barsh, G.S., Rauvala, H. and Bernfield, M. (2001) Transgenic expression of syndecan-1 uncovers a physiological control of feeding behavior by syndecan-3. Cell, 106, 105-116. doi:10.1016/S0092-8674(01)00415-9

[10] Lauri, S.E., Kaukinen, S., Kinnunen, T., Ylinen, A., Imai, S., Kaila, K., Taira, T. and Rauvala, H. (1999) Reg1ulatory role and molecular interactions of a cellsurface heparan sulfate proteoglycan (N-syndecan) in hippocampal long-term potentiation. The Journal of Neuroscience, 19, 1226-1235.

[11] Kaksonen, M., Pavlov, I., Võikar, V., Lauri, S.E., Hienola, A., Riekki, R., Lakso, M., Taira, T. and Rauvala, H. (2002) Syndecan-3-deficient mice exhibit enhanced LTP and impaired hippocampus-dependent memory. Molecular and Cellular Neuroscience, 21, 158-172. doi:10.1006/mcne.2002.1167

[12] Heldin, C.H. (1995) Dimerization of cell surface recaptors in signal transduction. Cell, 80, 213-223. doi:10.1016/0092-8674(95)90404-2

[13] Schlessinger, J. (2002) Ligand-induced, receptor-mediated dimerization and activation of EGF receptor. Cell, 110, 669-672. doi:10.1016/S0092-8674(02)00966-2

[14] Lemmon, M.A. and Schlessinger, J. (1994) Regulation of signal transduction and signal diversity by receptor oligomerization. Trends in Biochemical Sciences, 19, 459463. doi:10.1016/0968-0004(94)90130-9

[15] Weiss, A. and Schlessinger, J. (1998) Switching signals on or off by receptor dimerization. Cell, 94, 277-280. doi:10.1016/S0092-8674(00)81469-5

[16] Choi, S., Lee, E., Kwon, S., Park, H., Yi, J.Y., Kim, S., Han, I.O., Yun, Y. and Oh, E.S. (2005) Transmembrane domain-induced oligomerization is crucial for the functions of syndecan-2 and syndecan-4. The Journal of Cell Biology, 280, 42573-42579. doi:10.1074/jbc.M509238200

[17] Asundi, V.K. and Carey, D.J. (1995) Self-association of $\mathrm{N}$-syndecan (syndecan-3) core protein is mediated by a novel structural motif in the transmembrane domain and ectodomain flanking region. The Journal of Biological Chemistry, 270, 26404-26410. doi:10.1074/jbc.270.44.26404

[18] Carey, D.J., Stahl, R.C., Tucker, B., Bendt, K.A. and Cizmeci-Smith, G. (1994) Aggregation-induced association of syndecan-1 with microfilaments mediated by the cytoplasmic domain. Experimental Cell Research, 214, 12-21. doi:10.1006/excr.1994.1228

[19] Lax, I., Mitra, A.K., Ravera, C., Hurwitz, D.R., Rubinstein, M., Ullrich, A., Stroud, R.M. and Schlessinger, J. (1991) Epidermal growth factor (EGF) induces oligomerization of soluble, extracellular, ligand-binding domain of EGF receptor. The Journal of Biological Chemistry, 266, 13828-13833.

[20] Sorkin, A., McClure, M., Huang, F. and Carter, R. (2000) Interaction of EGF receptor and grb2 in living cells visualized by fluorescence resonance energy transfer (FRET) microscopy. Current Biology, 10, 1395-1398. doi:10.1016/S0960-9822(00)00785-5

[21] Youvan, D.C., Silva, C.M., Bylina, E.J., Coleman, W.J., 
Dilworth, M.R. and Yang, M.M. (1997) Calibration of fluorescence resonance energy transfer in microscopy using genetically engineered GFP derivatives on nickel chelating beads. Biotechnology et Alia, 3, 1-18.

[22] Hamdan, F.F., Audet, M., Garneau, P., Pelletier, J. and Bouvier, M. (2005) High-throughput screening of G protein-coupled receptor antagonists using a bioluminescence resonance energy transfer 1-based beta-arrestin2 recruitment assay. Journal of Biomolecular Screening, 10, 463-475. doi:10.1177/1087057105275344

[23] Angers, S., Salahpour, A., Joly, E., Hilairet, S., Chelsky, D., Dennis, M. and Bouvier, M. (2000) Detection of beta 2-adrenergic receptor dimerization in living cells using bioluminescence resonance energy transfer (BRET). Proceedings of the National Academy of Sciences of the United States of America, 97, 3684-3689.

[24] Heding, A. (2004) Use of the BRET 7TM receptor/ beta-arrestin assay in drug discovery and screening. Expert Review of Molecular Diagnostics, 4, 403-411. doi:10.1586/14737159.4.3.403

[25] Blanquart, C., Gonzalez-Yanes, C. and Issad, T. (2006) Monitoring the activation state of insulin/insulin-like growth factor-1 hybrid receptors using bioluminescence resonance energy transfer. Molecular Pharmacology, 70, 1802-1811. doi:10.1124/mol.106.026989

[26] Barnett, M.W., Fisher, C.E., Perona-Wright, G. and Davies, J.A. (2002) Signalling by glial cell line-derived neurotrophic factor (GDNF) requires heparan sulphate glycosaminoglycan. Journal of Cell Science, 115, 44954503. doi: $10.1242 /$ jes. 00114

[27] Sariola, H. and Saarma, M. (2003) Novel functions and signalling pathways for GDNF. Journal of Cell Science,
116, 3855-3862. doi: $10.1242 /$ jcs.00786

[28] Asundi, V.K., Erdman, R., Stahl, R.C. and Carey, D.J. (2003) Matrix metalloproteinase-dependent shedding of syndecan-3, a transmembrane heparan sulfate proteoglycan, in Schwann cells. Journal of Neuroscience Research, 73, 593-602. doi:10.1002/jnr.10699

[29] Berndt, C., Casaroli-Marano, R.P., Vilaró, S. and Reina, M. (2001) Cloning and characterization of human syndecan-3. Journal of Cellular Biochemistry, 82, 246-259. doi:10.1002/jcb.1119

[30] Carey, D.J., Stahl, R.C., Cizmeci-Smith, G. and Asundi, V.K. (1994) Syndecan-1 expressed in Schwann cells causes morphological transformation and cytoskeletal reorganization and associates with actin during cell spreading. The Journal of Cell Biology, 124, 161-170. doi:10.1083/jcb.124.1.161

[31] Bass, M.D. and Humphries, M.J. (2002) Cytoplasmic interactions of syndecan-4 orchestrate adhesion receptor and growth factor receptor signalling. Biochemical Journal, 368, 1-15. doi:10.1042/BJ20021228

[32] Filla, M.S., Woods, A., Kaufman, P.L. and Peters, D.M. (2006) Beta1 and beta3 integrins cooperate to induce syndecan-4-containing cross-linked actin networks in human trabecular meshwork cells. Investigative Ophthalmology \& Visual Science, 47, 1956-1967. doi:10.1167/iovs.05-0626

[33] Choi, Y., Kim, S., Lee, J., Ko, S.G., Lee, W., Han, I.O., Woods. A. and Oh, E.S. (2008) The oligomeric status of syndecan-4 regulates syndecan-4 interaction with alpha-actinin. European Journal of Cell Biology, 87, 807815. doi:10.1016/j.ejcb.2008.04.005 


\section{SUPPLEMENTARY MATERIAL}

Table S1. Methods used to study homo- or heterodimerisation of syndecans.

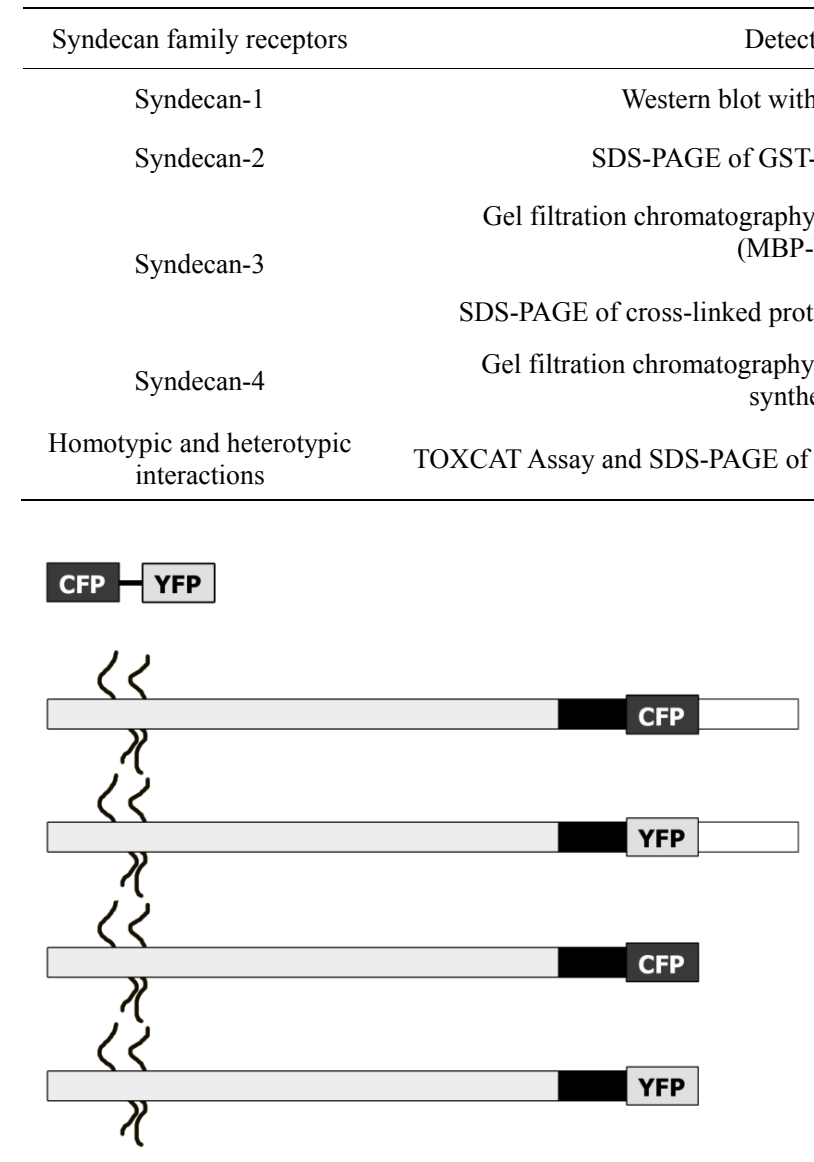

(a)

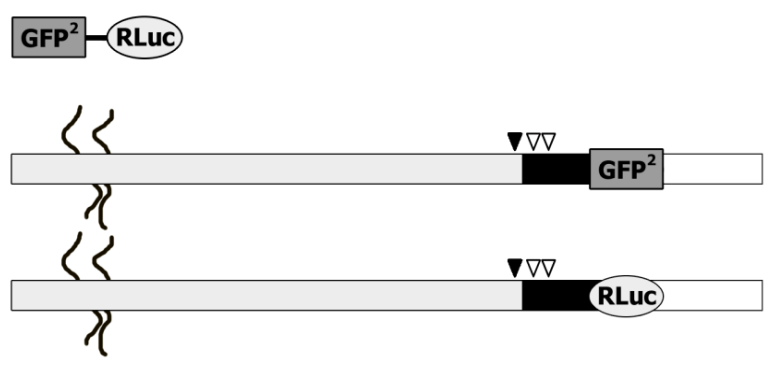

(b)

Figure S1. Schematic representation of N-syndecan fusion constructs. (a) Constructs used in FRET-imaging experiments. CFP and YFP were linked right after the transmembrane part (shown as a black rectangle) of N-syndecan. The extracellular region (with heparan sulfate chains) and the cytoplasmic tail are shown as grey and unfilled rectangles respectively. (b) Nsyndecan-GFP2/Rluc fusion proteins that were used in BRET2 assay experiments. Mutations of $\mathrm{N}$-syndecan are shown by arrowheads; K383A in juxtamembrane is depicted as black arrowhead and the mutations in the transmembrane part (G392L, G396L) are represented by white arrowheads.

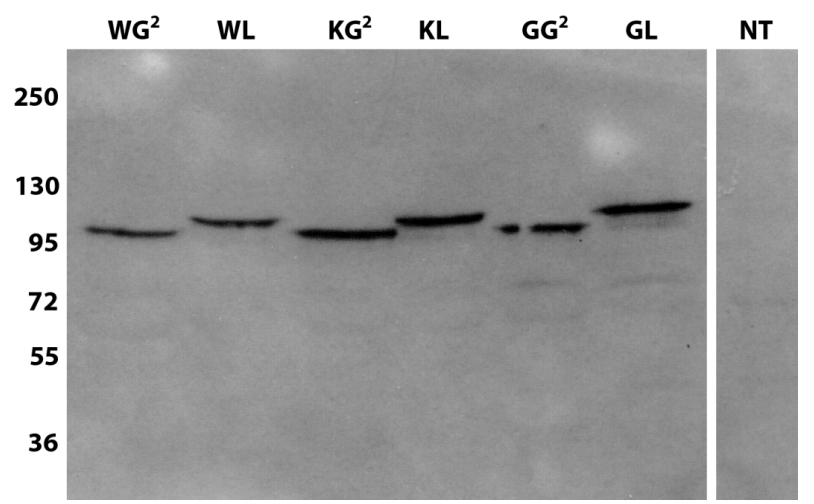

(a)
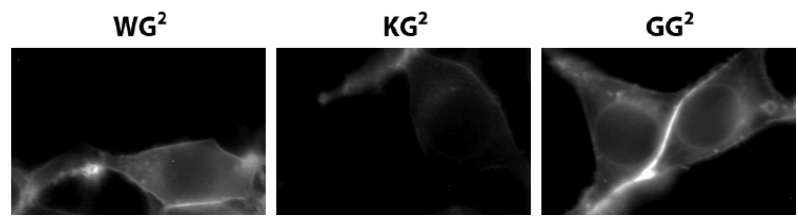

(b)

Figure S2. (a) Western-blot analysis of whole cell lysates of HEK $293 \mathrm{~T}$ cells transfected with syndecan mutants using anti N-syndecan antibodies (sc-9496, Santa Cruz). Both mutant variants are expressed at similar level compared to the wild type protein. (b) Control of localization by fluorescent imaging of GFP-fused mutants and wild-type N-syndecan. $\mathrm{WG}^{2}$, WLwild type $\mathrm{N}$-syndecan fusion proteins with $\mathrm{GFP}^{2}$ or Renilla luciferase (Rluc); KG2, KL-K383A mutants of N-syndecan fused with $\mathrm{GFP}^{2}$ and luciferase; $\mathrm{GG}^{2}$, GL-G392L mutants of $\mathrm{N}$-syndecan fused with $\mathrm{GFP}^{2}$ and luciferase. 